\title{
Giant Hexagonal Superstructures in Diblock-Copolymer Membranes
}

\author{
Christopher K. Haluska, ${ }^{1}$ Wojciech T. Góźdź, ${ }^{2,4}$ Hans-Günther Döbereiner, ${ }^{1, *}$ Stephan Förster, ${ }^{3}$ and Gerhard Gompper ${ }^{4}$ \\ ${ }^{1}$ Max-Planck-Institut für Kolloid- und Grenzflächenforschung, Am Mühlenberg 1, 14476 Golm, Germany \\ ${ }^{2}$ Institute of Physical Chemistry, Polish Academy of Sciences, Kasprzaka 44/52, 01-224 Warsaw, Poland \\ ${ }^{3}$ Institut für Physikalische Chemie, Universität Hamburg, Bundesstrasse 45, 20145 Hamburg, Germany \\ ${ }^{4}$ Institut für Festkörperforschung, Forschungszentrum Jülich, D-52425 Jülich, Germany
}

(Received 15 May 2002; published 18 November 2002)

\begin{abstract}
We have observed polymersomes of high genus with their vesicle wall organized on the micrometer scale either in a double bilayer connected by a lattice of passages or a tubular network with hexagonal symmetry. Experimentally found shape classes are identified within a theoretical phase diagram based on the bending energy of the polymer membrane. Pronounced morphological changes could be induced and controlled by temperature.
\end{abstract}

DOI: $10.1103 /$ PhysRevLett.89.238302

PACS numbers: 82.70.-y, 68.15.+e, 83.80.Uv, 87.16.Dg

Controlled structuring of materials on all length scales is required to tune their specific chemical and physical properties. In this respect, the complex molecular organization of block copolymer melts has been the focus of intense efforts over the last two decades [1]. Adding solvent to amphiphilic block copolymers melts produces an even richer zoo of marvelous morphologies on a supramolecular scale of 10 to $100 \mathrm{~nm}[2,3]$. Finally, advances are being made to extend structuring into the micrometer domain [4,5]. Recently, giant bilayer vesicles with a typical size of $10 \mu \mathrm{m}$ made of a homogenous diblockcopolymer membrane, so-called polymersomes, could be obtained in aqueous solution [6]. In this Letter, we report on the remarkable property of polybutadiene (32)- $b$-polyethylenoxide (20) (PB-PEO) molecules [7] to self-assemble into giant vesicles with a wall formed either by a double bilayer, which is connected by a lattice of passages, or a tubular network with hexagonal symmetry. The passages form the dual lattice of the hexagonal tubular network. In Fig. 1, we show an example of the generic morphology. We identify the bending elastic energy of the polymer membrane as the underlying mechanism of the observed microstructure formation, where the occurrence of curved interfaces is not directly linked to the nanoscale molecular architecture and alignment.

Lipid-bilayer vesicles with a small genus $g$, i.e., number of handles, have been investigated before for $g=1[8,9]$ and $g=2,4[10,11]$, where for $g>1$ conformal diffusion in vesicle shape space is observed [10,11]. In contrast, the vesicles we consider here have a high-genus topology, with a genus on the order of $g \simeq 100$ or even larger. We are not interested in global vesicle shapes [12,13], but rather want to characterize and model the local membrane shape on the scale of a typical structural unit.

The viscoelastic properties of a PB-PEO bilayer membrane have already been investigated in Ref. [14]. Using micropipette aspiration of giant quasispherical vesicles (with genus $g=0$ ), the bending and stretching elastic moduli of the polymer membrane were found to be $\kappa=$
$42 k_{B} T$ and $K=470 \mathrm{dyn} / \mathrm{cm}$, respectively. These values fall in the range common to lipid membranes. These findings parallel an earlier study in aqueous solution of the very similar diblock-copolymer polyethylethylene (37)- $b$-polyethylene oxide (40) [6]. Further, rheological experiments show the PB-PEO membrane to be fluid [14]. However, the membrane viscosity is increased almost 1000 -fold as compared to typical lipid membranes. Nevertheless, at room temperature one is favorably above the glass temperature of the short-chain polybutadiene used in this study. Thus, as in the case of lipid membranes, we may model the block copolymer membrane as a fluid elastic sheet allowing bending deformations, but

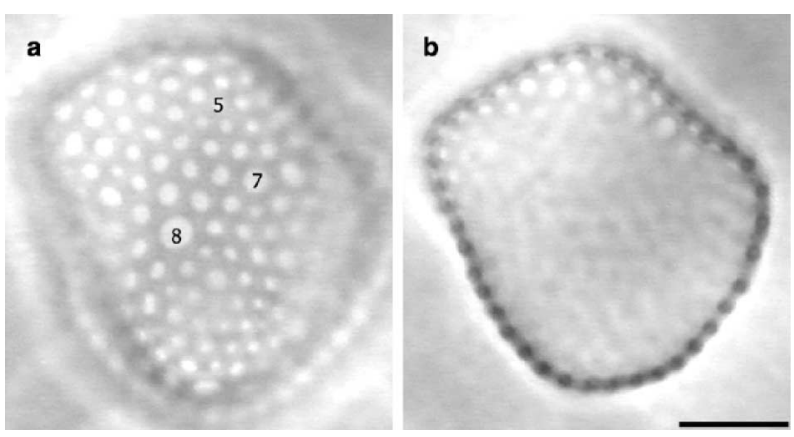

FIG. 1. High genus polymersome in glucose solution. The top view (a) shows a phase contrast micrograph of a typical hexagonal lattice of passages in a double bilayer vesicle surface. The space between the membranes is filled with sucrose solution inducing a dark contrast. The passages between the core volume and the outside of the vesicle appear in light contrast due to the continous space filled with glucose solution. Topologically, the vesicle is single connected with a large number of handles. Note the two larger passages forming sevenfold and eightfold coordination defects together with a fivefold coordinated passage. The cross section (b) of the same vesicle shows nearly circular tubes (dark contrast) forming the double bilayer surface. The scale bar corresponds to $10 \mu \mathrm{m}$. 
essentially no area dilatation due to the high stretching elastic modulus.

Vesicles are swollen in sucrose solution and incubated in glucose solution of matched osmolarity $(100 \mathrm{mMol})$ in order to adjust vesicle density in the sample and to enhance contrast for observation under a phase contrast microscope (Zeiss Axiovert 135, 40× Ph2). We note that high-genus structures were already present before addition of glucose solution. Typically, samples were monitored within a few days after preparation. Occasionally, we observed samples several weeks later and could not find a significant difference in appearance. Samples were examined in a temperature controlled $\left( \pm 0.1^{\circ} \mathrm{C}\right)$ microchamber which was tightly sealed to avoid evaporation and deflation of vesicles. Appropriate vesicles exhibiting a microstructured membrane were selected for observation and monitored for several hours at various temperatures. We examined more than 20 vesicles in detail. Hundreds of additional high-genus structures were seen but not monitored over time. Often these polymer structures were clumped together or exhibited an unresolvable topology not suitable for quantitative analysis. We found a wide distribution of vesicle genus with predominantly spherical topology. Only a small number of well-defined highgenus vesicles per chamber could be found. However, the structures observed were always present and proved robust against variations in preparation conditions. In fact, we could swell high-genus vesicles in $\mathrm{NaCl}$ solutions (1 m Mol, $100 \mathrm{~m} \mathrm{Mol}$ ) as well.

Our theoretical analysis of the local membrane shapes of these high-genus polymersomes is based on the curvature energy of doubly periodic membranes with the constraints of fixed volume $V$ and membrane area $S$. The walls of the polymersomes, which are formed of a double bilayer connected by passages, can be well approximated by doubly periodic surfaces when the distance between the bilayers is small compared to the size of the polymersomes. We study a lattice of passages of hexagonal symmetry, since this is the local symmetry most often observed experimentally. For simplicity, we ignore the existence of lattice defects, in particular, those which are induced by the spherical topology of the polymersome shapes, see Fig. 1. Membrane shapes with passages, which are symmetric with respect to the midplane between the two bilayers, can be parametrized by $[15,16]$

$$
\cosh ^{2}(z)=a_{0}+\sum_{i=1}^{N} a_{i} \sum_{j=1}^{N_{i}} \cos \left[\frac{2 \pi}{L} \mathbf{k}_{j}^{(i)} \cdot \mathbf{r}\right],
$$

where $\mathbf{r}=(x, y)$, and $N$ is the number of Fourier amplitudes. There are $N_{i}$ reciprocal lattice vectors $\mathbf{k}^{(i)}$ in the $i$ th shell. $L$ is the lattice constant. No $\sin \left[\mathbf{k}_{j}^{(i)} \cdot \mathbf{r}\right]$ terms appear in the expansion (1) because we assume the full symmetry of the hexagonal lattice. The periodic boundary conditions account for the fact that the polymersomes are closed, so that there are no free edges.
The elastic properties of diblock-copolymer bilayers are described, as for lipid bilayers, by the curvature energy [17],

$$
\mathcal{H}=\frac{\kappa}{2} \int d S\left(C_{1}+C_{2}-2 C_{0}\right)^{2},
$$

where the integral extends over the whole membrane area. $C_{1}, C_{2}$ are the local principal curvatures at each point of the membrane. The local membrane shape of polymersomes indicates that the diblock bilayer must have nonzero spontaneous curvature $C_{0}$. The spontaneous curvature is induced by the different sugar solutions on the two sides of the polymer membrane [18]. The two sugar species are likely to interact in a different way with the PEO chains of the respective diblock copolymer, e.g., via competition for hydration. A second contribution to the spontaneous curvature arises from the different number of polymer molecules in the two sheets of the bilayer, which typically occurs during the formation process. As a consequence, absolute temperature is not significant to vesicle shapes. What is important are changes in temperature which induce a shift in spontaneous curvature. Our experimental evidence suggest that the bilayers composing a polymersome do not break, and the topology of the membrane does not change; a Gaussian-curvature term $C_{1} C_{2}$ is therefore omitted from Eq. (2). A related problem, with vanishing spontaneous curvature $C_{0}=0$, variable topology, square symmetry, and fixed lattice constant, has been considered theoretically in Ref. [19].

We examine the stability of local membrane shape as a function of dimensionless variables $v=V / S^{3 / 2}$ and $c_{0}=$ $C_{0} S^{1 / 2}$. Here, volume $V$ and membrane area $S$ are calculated for the unit cell of the hexagonal lattice. We have found several families of shapes: small and large passages, budded vertices, tubes, and spindles, which are stable for different values of $v$ and $c_{0}$. Experimental membrane morphologies can be matched nicely with their respective corresponding theoretical counterparts, as demonstrated in Fig. 2. The regions of stability of these phases can be seen in the phase diagram of Fig. 3.

The most common shapes are membranes connected by circular passages. For $v \lessgtr 0.10$, shapes of two families of circular passages exist for the same values of $v$ and $c_{0}$ over some range of spontaneous curvature. The shapes of these two families differ mainly by the size of the passage, where one family - see Figs. 2(a) and 2(b) - is characterized by a small ratio of passage radius to lattice constant, while the other-see Figs. 2(c) and 2(d) - is characterized by a large ratio. The "small-passage" shapes are most stable for low values of $c_{0}$, while the "large-passage" shapes are the most stable for larger values of $c_{0}$. The transition from small to large passages with increasing $c_{0}$ is discontinuous for small reduced volume $v$, and ends at a critical point. The transition is accompanied by the change of the lattice constant $L$ of 

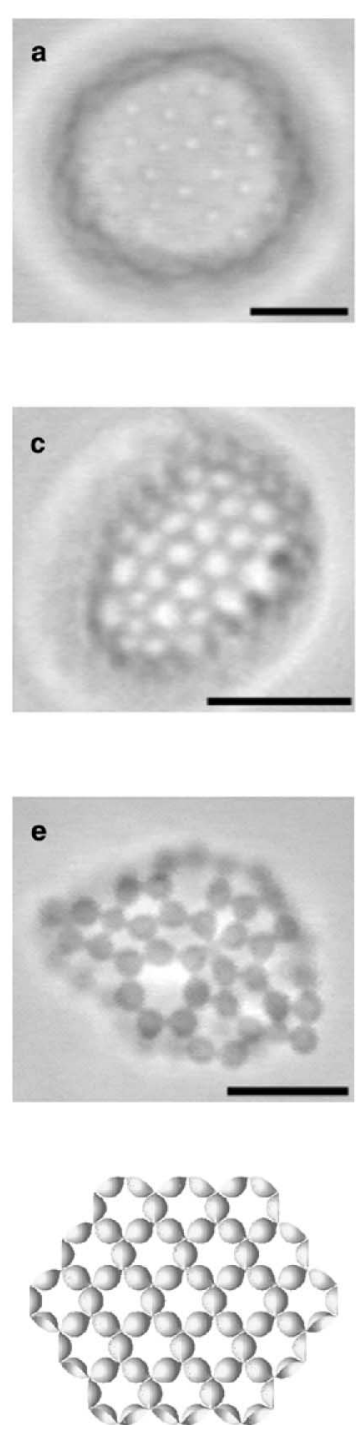

g

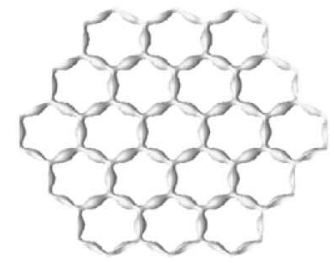

FIG. 2. Comparison of experimental and theoretical shapes. The scale bars correspond to $10 \mu \mathrm{m}$. Experimental images with (a) small passages, (c) large passages, (e) budded vertices. Theoretical shapes resulting from minimization of the functional (2) with $N=15$ in Eq. (1) for (b) $v=V / S^{3 / 2}=0.098$ and $c_{0}=C_{0} S^{1 / 2}=0.3$ (small passages), (d) $v=0.076$ and $c_{0}=2.7$ (large passages), (f) $v=0.065$ and $c_{0}=4.4$ (budded vertices), $(\mathrm{g}) v=0.053$ and $c_{0}=5.7, v=0.043$ and $c_{0}=5.9$ (spindles). The lattice of touching spheres (e) is deformed by strong thermal fluctuations.

the order of a few percent. The region in the phase diagram, in which solutions for both small and large passages exist, is marked by a shaded area in Fig. 3. Shapes, which resemble spheres located on the vertices of the hexagonal lattice connected by small necks, are stable for a range of reduced volumes $0.051 \lesssim v \lesssim 0.071$ and for $c_{0} \gtrsim 3.9$, as shown in Figs. 2(e) and 2(f). For spontaneous curvature $c_{0}=\sqrt{8 \pi}$ and reduced volume $v=1 /(6 \sqrt{2} \bar{\pi})$, these shapes degenerate into touching spheres. The apparent lack of symmetry in the experimental Fig. 2(e) compared to its theoretical counterpart

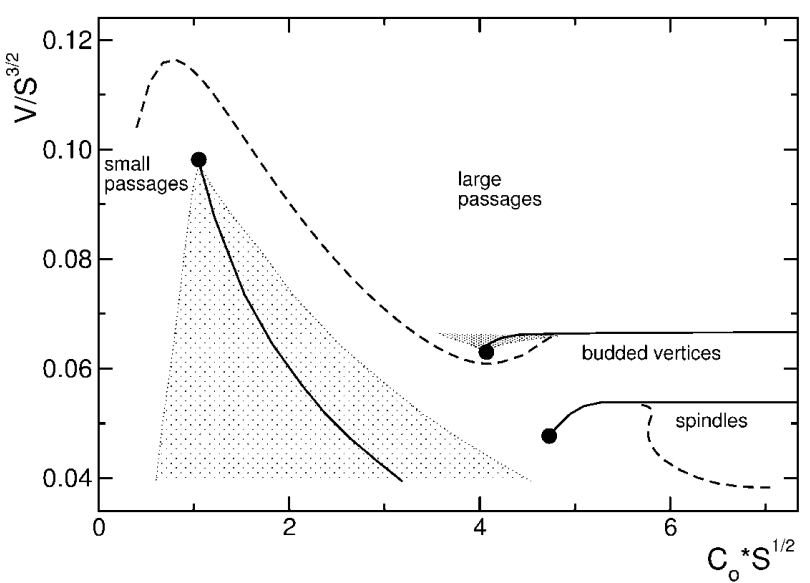

FIG. 3. Theoretical phase diagram, calculated for $N=15$ Fourier amplitudes in Eq. (1). The full lines indicate phase transitions, where the energies of two different families of shapes are equal. All transitions are first-order and end with criticial points $(\bullet)$. The dashed lines are locations of minimal energy when the volume constraint is released. The shaded areas show the regions where shapes of two different families are (meta)stable.

Fig. 2(f), is likely caused by a small energy on the order of $k_{B} T$ required to deform the lattice of touching spheres. For smaller values of $v$, the spheres stretch out towards their nearest neighbors. With increasing spontaneous curvature, the "large-passage" phase transforms smoothly into these "budded vertices." Only very close to the value of $v$ for touching spheres does the transition become discontinuous. The line of discontinuous transitions ends at a "lower critical point" when $v$ is decreased.

Further decreasing of $v$ leads to yet another family of shapes, which are composed of a spindlelike object located along the edges of a hexagonal lattice and connected at the vertices as shown in Fig. 2(g). The transformation of passages into spindles with increasing $c_{0}$, occurs via shapes, which are narrow tubes along the edges of the hexagonal network. There is a line of firstorder transitions for $0.047 \lesssim v \lesssim 0.055$, which ends again in a lower critical point. The boundaries of the metastable regions are not shown in Fig. 3 in this case. For $v \lesssim 0.047$, the family of large passages merges smoothly with the family of spindles. Unfortunately, we have not been able to observe regular patterns composed of the spindles experimentally; however, locally such shapes appear in polymersomes. The regions of stability for budded vertices and spindles have upper limits in reduced volume resulting from geometric constraints. Larger values of $v$ would lead to self-intersecting shapes in these families, which cannot be described by our ansatz (1).

In Fig. 2, we show only vesicles with a global prolate shape, although other more complicated shapes [13], e.g., starfish vesicles, have been found as well. In addition, we observed various shape transitions, e.g., budding of the 


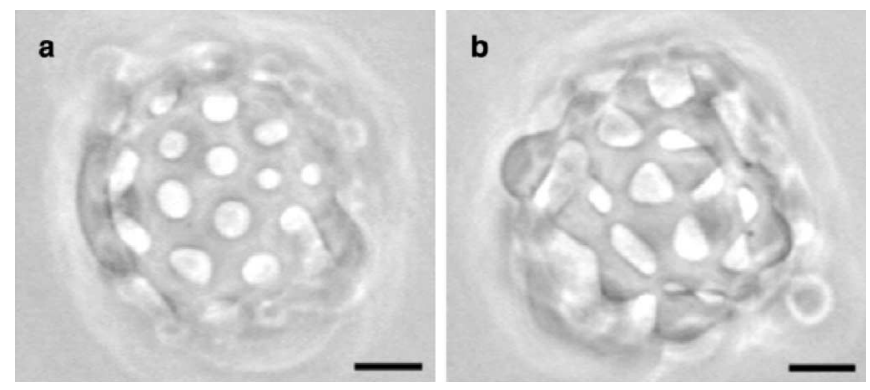

FIG. 4. Temperature induced transformation from (a) "large passages" to (b) "budded vertices." After a quench by $4 \mathrm{~K}$, the vesicle [(a) $T=45.7 \rightarrow 41.7 \mathrm{C}, t=0 \mathrm{~min}$ ] first shows budding of its vertices [(b) $41.7 \mathrm{C}, t=20 \mathrm{~min}$ ] before it relaxes back to its original large-passages shape (not shown; $41.7 \mathrm{C}$, $t=80 \mathrm{~min})$. The scale bars correspond to $10 \mu \mathrm{m}$.

microstructured vesicles. Transitions between distinct classes could be induced by temperature. Decreasing temperature progressively leads to double membrane structures with higher curvatures, see Fig. 4. Because of the high viscosity of the polymer membrane and the resulting slow vesicle fluctuations and membrane relaxation, we could not decide whether the transition between the "passage" and the "budded vertices" class is of first or second order. Generally, transitions appeared to be continuous. The vesicle shown in Fig. 4(b) exhibited a predominantly triangular symmetry in the budded-vertex state. In some cases, we also observed regions with square symmetry on the scale of several unit cells. These symmetry classes are probably low lying metastable states rather than stable membrane shapes, since the vesicle relaxed back to its original shape [Fig. 4(a)] at constant temperature within about $1 \mathrm{~h}$. Remarkably, the same cycle of shape changes could be induced several times upon further lowering the temperature in jumps of a few degrees. After each jump, a transformation to the budded-vertex state is followed by a relaxation back to the large-passage state. At higher temperatures, enhanced membrane fluctuations are found, which indicate a decrease in membrane viscosity, as might be expected.

To conclude, we have shown that diblock-copolymer membranes can exhibit regular superstructures on a micrometer scale. We found a pronounced morphological response to changes in spontaneous curvature, which allows a precise control of the shape of the pores in the double-bilayer membrane. In addition to temperature, there are other natural control parameters of interfacial shape, such as electrolyte concentration and $p \mathrm{H}$ as well as the degree of hydrogen bonding, which will be effective via their influence on PEO chain conformations in solution. We could control the morphology of polymersomes with fixed topology. In contrast, the detailed molecular mechanisms of high-genus vesicle formation and the distribution of genus remains to be understood. Structuring polymeric interfaces on micrometer length scales may prove useful for applications requiring materials with giant pores. In a biomimetic context, we note the striking resemblance of the large-passage membrane structure Figs. 2(c) and 2(d) to the tests of certain sea urchins [20], which use syncytial membranes to direct growth of their mineral skeleton. Albeit this biological controlled inorganic morphosynthesis [21] is far from being completely understood, it seems promising to employ superstructures of diblock-copolymer membranes in order to cast desired mineral materials into micron-sized geometrical shapes.

We thank Antje Reinecke for technical assistance and help in part of the experiments. H.-G. D. thanks Udo Seifert for enjoyable and helpful discussions. W. T. G. acknowledges support from the Komitet Badań Naukowych (KBN 7T09A07821) and from the Deutscher Akademischer Austauschdienst (DAAD).

*Electronic address: hgd@mpikg-golm.mpg.de

[1] F. S. Bates and G. H. Fredrickson, Phys. Today 52, No. 2, 32 (1999).

[2] N. S. Cameron, M. K. Corbierre, and A. Eisenberg, Can. J. Chem. 77, 1311 (1999).

[3] P. Alexandridis and R. J. Spontak, Curr. Opin. Collod Interface Sci. 4, 130 (1999).

[4] Y.-Y. Won, H.T. Davis, and F. S. Bates, Science 283, 960 (1999).

[5] J. N. Cha, G. D. Stucky, D. E. Morse, and T. J. Deming, Nature (London) 403, 289 (2000).

[6] B. M. Discher et al., Science 284, 1143 (1999).

[7] S. Förster and E. Krämer, Macromolecules 32, 2783 (1999).

[8] U. Seifert, Phys. Rev. Lett. 66, 2404 (1991).

[9] B. Fourcade, M. Mutz, and D. Bensimon, Phys. Rev. Lett. 68, 2551 (1992).

[10] F. Jülicher, U. Seifert, and R. Lipowsky, Phys. Rev. Lett. 71, 452 (1993).

[11] X. Michalet and D. Bensimon, Science 269, 666 (1995).

[12] R. Lipowsky, Nature (London) 349, 475 (1991).

[13] H.-G. Döbereiner, in Giant Vesicles, edited by P. L. Luisi and P. Walde (John Wiley \& Sons, New York, 2000), p. 150 .

[14] R. Dimova, U. Seifert, B. Pouligny, S. Förster, and H.-G. Döbereiner, Eur. Phys. J. E 7, 241 (2002).

[15] W.T. Góźdź and G. Gompper, Phys. Rev. Lett. 80, 4213 (1998).

[16] W.T. Góźdź and G. Gompper, Phys. Rev. E 59, 4305 (1999).

[17] W. Helfrich, Z. Naturforsch. 28c, 693 (1973).

[18] H.-G. Döbereiner, O. Selchow, and R. Lipowsky, Eur. Biophys. J. 28, 174 (1999).

[19] T. Charitat and B. Fourcade, J. Phys. II (France) 7, 15 (1997).

[20] K. Simkiss and K. M. Wilbur, Biomineralization (Academic Press, New York, 1989).

[21] S. Mann and G. A. Ozin, Nature (London) 382, 313 (1996). 